\title{
Follow-up of skin lesions during COVID-19: a description of a DRESS case
}

\author{
Alexandra Maria Giovanna Brunasso ${ }^{1}$ (1) - Marco Castellaneta ${ }^{2} \cdot$ Emanuele Pontali $^{3} \cdot$ Francesca Raggi $^{2}$. \\ Cesare Massone ${ }^{1}$
}

Received: 22 June 2020 / Published online: 6 January 2021

○) Springer-Verlag GmbH Germany, part of Springer Nature 2021

Keywords COVID-19 $\cdot$ Skin $\cdot$ SARS-CO-V-2 $\cdot$ Exanthema $\cdot$ Eosinophilia $\cdot$ DRESS

To the Editor,

We read with interest the paper by De Medeiros et al. regarding the follow-up of skin lesions during COVID-19; they suggest that we must pay attention to other aetiologies such as reactivation of other viral coexistent infections or other factors. We would like to add another report to confirm that some cutaneous manifestations during COVID-19 are secondary and not directly related to SARS-CoV-2, demonstrating that it might only be a bystander in a critical illness where indirect immune injury plays an important role and the use of multiple medications is required. We observed a 50-year-old female who developed abrupt onset of fever, morbilliform exanthema ( $80 \%$ of BSA) with severe pruritus and facial oedema after 34 days from COVID-19 onset of symptoms (day-0) and after 26 days of intensive care unit (ICU) admission (Fig. 1). Detailed laboratory investigations during the last 40 days of admission are shown in Fig. 2. Ten days (day-24) prior to the onset of the exanthema, levofloxacin $500 \mathrm{mg}$ bid i.v was introduced due to Klebsiella aerogenes nosocomial infection. At the onset of the exanthema (day-34), eosinophils count started to increase (peak of $5.73 \times 10^{9}$ cells $\left./ \mathrm{L}\right)$ associated with lymphocytosis $\left(3.48 \times 10^{9}\right.$

This comment refers to the article available online at https://doi. org/10.1007/s00403-020-02091-0.

Alexandra Maria Giovanna Brunasso

alexandra.brunasso@galliera.it

1 Department of Dermatology, Galliera Hospital, Via Volta, 6, 16128 Genoa, Italy

2 Department of Internal Medicine, Galliera Hospital, Genoa, Italy

3 Department of Infectious Diseases, Galliera Hospital, Genoa, Italy
cells/L), increase of protein-C-reactive (28.7 mg/L) and alanine transaminase (63 U/L) and normal creatinine (Fig. 2). Mediastinal lymph node enlargement, persistent interstitial pneumonia and liver enlargement were seen on control computer tomography after the onset of the exanthema. The diagnosis of levofloxacin-induced DRESS during COVID19 was made. Histopathological examination was not performed due to COVID-19 isolation measures. Fever, liver involvement (quickly) and skin lesions (slowly) improved within 3 weeks after the discontinuation of levofloxacin and the beginning of systemic (prednisone $1 \mathrm{mg} / \mathrm{kg} /$ day i.v) and topical corticosteroids. The causality between DRESS and levofloxacin in our case can be described as probable because rechallenge cannot be conducted due to the high mortality rate probability $(10 \%)$ [2-4]. DRESS is a rare, life-threatening, non-dose related and unpredictable adverse drug reaction [2-4]. Diagnostic criteria are cutaneous exanthema covering more than 50\% BSA, eosinophilia $>1500 / \mathrm{L}$ and/or lymphocytosis, lymph node enlargement $>2 \mathrm{~cm}$, hepatitis (transaminase $>2$ normal levels), interstitial pneumonia, carditis and interstitial nephritis [2-4]. DRESS develops in 1 week up to 3 months after a new drug assumption [2-4]. Various drugs can trigger DRESS, especially aromatic anticonvulsants and sulphonamides, but other medications have been reported as terbinafine, ramipril, metronidazole, minocycline and levofloxacin [2-4]. It has also been suggested a concomitant reactivation of $\mathrm{HHV}-6, \mathrm{HHV}-7, \mathrm{CMV}$, and EBV that may play a role [1-4]. 


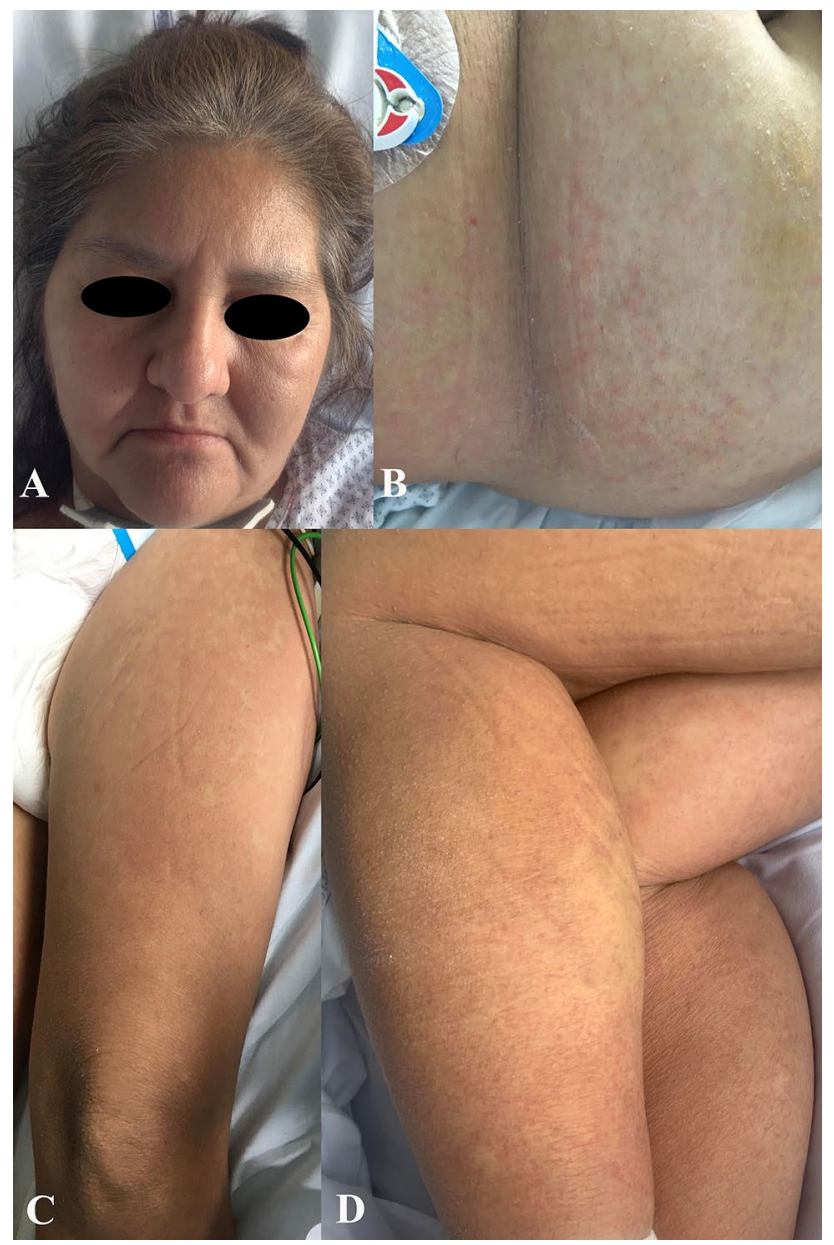

Fig. 1 Clinical findings: a Facial edema. Morbilliform exanthema with follicular accentuation on the left flank (b), left thigh (c) and both legs (d)
Several evidences speak against a causative role of SARS$\mathrm{CoV}-2$ in late-onset exanthema. Patients affected by severe COVID-19 require prolonged hospitalizations, introduction of several new medications and frequently develop nosocomial infections; in such a context, skin drug reactions might be expected [5]. The exanthema to be COVID-19 specific (directly related to the virus) requires haematogenous viral spread through the cutaneous vascular system [6]. SARSCoV-2 RNAaemia (viraemia) has been detected only in few critically ill-patients $[7,8]$. Until today, we were not able to find reports of skin manifestations with SARS-CoV-2-RNA detection in blood or in skin material. On the other hand, viral reactivation of Parvovirus B-19 and EBV, as well as drug-related-exanthemas, mainly in less severe COVID-19 patients can be found as suggested by de Medeiros et al. [1, $9,10]$. The onset of exanthema was delayed in the course of COVID-19 (during the recovery phase) and not in the initial acute stage when we can expect an RNAaemia with cutaneous vascular involvement. Drug anamnesis should be carefully investigated in COVID-19 patient with exanthema. 
Fig. 2 Laboratory finding during hospital admission: initial lymphopenia, leukocytosis, elevated D-dimer and reactiveC-protein due to severe COVID19. After 26 days of ICU admission, onset of exanthema with marked eosinophilia, lymphocytosis, increase of alanine transaminase (ALT: $>2$ normal levels), D-dimer and reactive $\mathrm{C}$ protein and improvement of all values after introduction of intravenous (i.v) prednisone

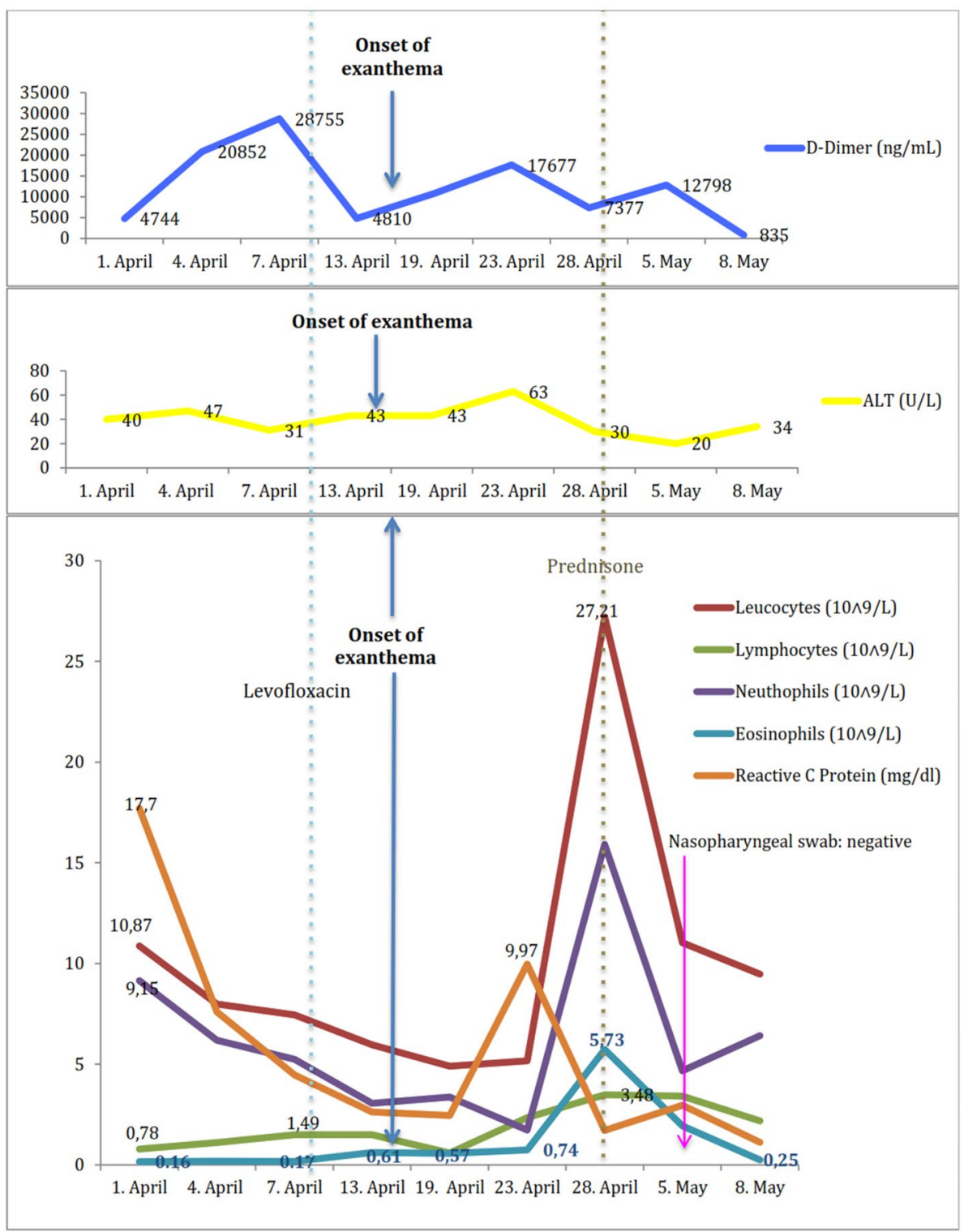

Acknowledgements The patient in this study has given written informed consent to publish their case details.

Funding None.

\section{Compliance with ethical standards}

Conflict of interest None declared.

\section{References}

1. de Medeiros VLS, Silva LFT (2020) Follow-up of skin lesions during the evolution of COVID-19: a case report. Arch Dermatol Res. https://doi.org/10.1007/s00403-020-02091-0
2. Pileri A, Brunasso AM, Tilz H, Wolf P, Massone C (2011) Ramipril-induced drug reaction with eosinophilia and systemic symptoms (DRESS). Eur J Dermatol 21:624-625

3. Matsumoto T, Horino T, Inotani S, Ichii O, Terada Y (2019) Drug reaction with eosinophilia and systemic symptoms/drug-induced hypersensitivity syndrome (DRESS/DIHS) caused by levofloxacin in a patient with systemic scleroderma, rheumatoid arthritis, and Sjogren syndrome. Contact Dermatitis 80:253-254

4. Mockenhaupt M. Drug reaction with eosinophilia and systemic symptoms (DRESS). In: UpToDate, Post TW (Ed), UpToDate, Waltham, MA. Accessed 7 June 2020

5. Zhou F, Yu T, Du R et al (2020) Clinical course and risk factors for mortality of adult inpatients with COVID-19 in Wuhan, China: a retrospective cohort. Lancet. 395:1054-1062

6. Gianotti R, Zerbi P, Dodiuk-Gad RP (2020) Clinical and histopathological study of skin dermatoses in patients affected by COVID-19 infection in the Northern part of Italy. J Dermatol Sci. https://doi.org/10.1016/j.jdermsci.2020.04.007 
7. Chen X, Zhao B, Qu Y et al (2020) Detectable serum SARSCoV-2 viral load (RNAaemia) is closely correlated with drastically elevated interleukin 6 (IL-6) level in critically ill COVID-19 patients. Clin Infect Dis. https://doi.org/10.1093/cid/ciaa449

8. Lescure FX, Bouadma L, Nguyen D et al (2020) Clinical and virological data of the first cases of COVID-19 in Europe: a case series. Lancet Infect Dis. https://doi.org/10.1016/S1473 $-3099(20) 30200-0$

9. Diaz-Guimaraens B, Dominguez-Santas M, Suarez-Valle A et al (2020) Petechial Skin Rash Associated With Severe Acute Respiratory Syndrome Coronavirus 2 Infection. JAMA Dermatol. https://doi.org/10.1001/jamadermatol.2020.1741.10.1001/jamad ermatol.2020.1741
10. Sanchez A, Sohier P, Benghanem S et al (2020) Digitate Papulosquamous Eruption Associated With Severe Acute Respiratory Syndrome Coronavirus 2 Infection. JAMA Dermatol. https:// doi.org/10.1001/jamadermatol.2020.1704.10.1001/jamadermat ol.2020.1704

Publisher's Note Springer Nature remains neutral with regard to jurisdictional claims in published maps and institutional affiliations. 